\title{
Cervical Prolapse During Pregnancy
}

\author{
Devin Sawyer, $M D$, and Keith Frey, $M D$
}

The occurrence of cervical prolapse during pregnancy is uncommon. Fewer than 245 cases have been reported in the literature, and only 5 have been reported since $1968 .{ }^{1-3}$ Most cases were reported before 1900. In $1941 \mathrm{Keettel}^{4}$ reported only 1 case in 13,000 deliveries, and in $1944 \mathrm{Kibel}^{5}$ reported a ratio of 1 in 15,696 deliveries. The incidence of uterine prolapse in India is much more common, estimated to be as high as 1 in 547 deliveries. ${ }^{6}$ There have been no cases of uterine prolapse reported in the family practice literature.

The reported complications that result from cervical prolapse during pregnancy have ranged from minor cervical desiccation and ulceration to devastating maternal fatalities. The scope of complications includes, but is not limited to, spontaneous abortion, preterm labor, premature delivery, fetal demise, and maternal sepsis. ${ }^{7}$ Keettel ${ }^{4}$ reported 14 cases of maternal sepsis with 8 deaths, the last of which occurred in 1925. With the advent of aseptic technique and antibiotics, the severity of maternal morbidity and rate of death have greatly diminished. Even so, Keettel ${ }^{4}$ and Piver and Spe$\mathrm{zia}^{8}{ }^{8}$ whose observations are separated by 27 years, reported similar rates of fetal death at $18.1 \%$ and $18.2 \%$, respectively. Patient discomfort, urinary tract infection, acute urinary retention, premature labor, and prenatal loss remain serious risks. ${ }^{7}$

Given that cervical prolapse is rare and has yet to be reported in the family practice literature, we have set out to cite a case and describe a rational, conservative approach to treatment that a family physician, with available obstetric backup, would feel comfortable practicing.

\section{Case Report}

A 31-year-old woman, gravida 2 para 1, sought initial prenatal care at 14 weeks' gestation. Her

Submitted, revised, 27 April 1999.

From Providence St. Peter Hospital Family Practice Residency Program (DS, KF), Olympia, Wash. Address reprint requests to Keith Frey, MD, Mayo Thunderbird Family Medicine Center, 13737 North 92nd St, Scottsdale, AZ 85260 . medical history was notable for a single abnormal Papanicolaou smear evaluated by colposcopy with negative biopsy findings. Between pregnancies, the patient underwent an elective, cosmetic abdominoplasty. Six years before her current pregnancy, she had an uncomplicated delivery of a full-term, 5 -pound 10-ounce infant. The patient is a hairdresser by occupation. Findings from her initial physical examination and laboratory evaluation were normal. A sonogram early in the second trimester confirmed her estimated gestational age.

At approximately $151 / 2$ weeks of gestational age, the patient came to the Emergency Department complaining of tissue prolapsing at the vaginal introitus. At that time fetal heart tones were confirmed at 150 beats per minute. A pelvic examination showed a low-lying closed cervix at the introitus. The findings from her physical examination were otherwise normal. The patient was cared for conservatively with bed rest and pelvic rest, with subsequent resolution of the prolapse within 24 hours. The rest of her pregnancy was unremarkable, and ongoing monitoring by cervical examinations showed no further prolapse, premature cervical dilation, or effacement. Screening for group B streptococcus at 36 weeks' gestation was negative. Labor was electively induced at $401 / 7$ weeks to insure adequate obstetric backup for the vaginal delivery. The patient's labor progressed normally through the first stage, and the second stage lasted a total of 12 minutes. The patient gave birth to a $3500-\mathrm{g}$ viable male infant with Apgars of 9 at 1 minute and 9 at 5 minutes. No prolapse of the uterus or cervix was noted during the second or third stage. A second-degree perineal laceration was repaired in the usual fashion. The placenta was delivered spontaneously.

Approximately 2 weeks postpartum the patient had a recurrence of her cervical prolapse. When examined, she had a slight protuberance at the introitus. Again, the patient was cared for conservatively, and at her 8-week postpartum examination, the uterus was normal in size, shape, and position, with no evidence of prolapse. 


\section{Discussion}

The main support of the uterus and vaginal vault is the Mackenrodt ligament, also known as the cardinal ligament. ${ }^{9}$ It forms a firm attachment from the supravaginal cervix to the tough obturator fascia on the side wall of the pelvis. Failure of these supportive ligaments leads to prolapse of the uterus and vaginal vault. Three factors lead to failure of the supportive ligaments and fasciae: ${ }^{9}$ childbirth trauma, congenital and developmental weakness, and the influence of menopause. Childbirth trauma is likely the cause of most reported cases of cervical prolapse complicating pregnancy. The gradual decrease in parity in the United States within the last several decades probably accounts for the recent low incidence of such occurrences. ${ }^{10}$ Cervical prolapse in nulliparous pregnant patients is likely to be secondary to congenital or developmental weakness of supportive fascia. ${ }^{3}$ Uterine prolapse in nonpregnant women is most common, however, in those who are past their child-bearing years, particularly during and after menopause. In addition, obesity, large pelvic inlet, and a history of difficult deliveries or large babies can predispose an otherwise supported uterus to fall through the introitus. ${ }^{1}$

Prolapse of the cervix during pregnancy might not be entirely secondary to simple uterine prolapse. Hypertrophic elongation of the cervix is also likely to contribute. ${ }^{6}$ As the pregnancy progresses through the fourth and fifth months, the uterus ascends out of the pelvis and the protruding cervix and prolapsed vaginal wall typically recede.

The association of cervical prolapse with pregnancy and abdominoplasty has not been well studied because of the rarity of such an occurrence. It is known that flaccidity of abdominal musculoaponeurotic tissues increases with subsequent pregnancies. ${ }^{11}$ Expert opinion suggests that abdominal relaxation and pelvic relaxation coexist, and by surgically repairing the former with abdominoplasty, the pelvic outlet could become the weakest area and the point through which increased abdominal pressure will transmit. ${ }^{12}$ Increased age and multiparity can further weaken the pelvic floor and predispose to cervical prolapse.

The treatment of cervical prolapse during pregnancy has ranged considerably, particularly in earlier times. Chinese native doctors saturated the protruding cervix with kerosene oil and set fire to it. $^{13}$ In 1911 Findley $^{13}$ recommended interruption of the pregnancy followed by plastic repair. Pessary placement was recommended as early as $1901 .^{14}$ From 1920 to 1945 the most common method of delivery was with forceps after Dührssen incisions. In 1968, however, Piver and Spezia ${ }^{8}$ reported a spontaneous delivery rate of $84.8 \%$, a considerable improvement from Keettel's reported $34.7 \% .^{4}$ Management options have not varied much in recent years. The key to successful treatment is early recognition with conservative management. Slight Trendelenburg position with bed rest and replacement of the uterus after resolution of edema will often protect the cervix from trauma and desiccation and decrease the incidence of preterm labor. ${ }^{1}$ Keeping the cervix moist is of great importance. Prophylactic antibiotics are not indicated. ${ }^{1}$ Placement of a well-fitting Smith-Hodge or doughnut pessary will often allow the patient to continue with the pregnancy without difficulty. ${ }^{7}$ Most patients will have spontaneous vaginal deliveries. If a pessary is used during pregnancy, it should be reinserted immediately postpartum to support the fascia and ligaments during involution. ${ }^{8}$

In summary, cervical prolapse predisposes the pregnant patient to many complications she would otherwise not face. The family physician who practices obstetrics should feel comfortable diagnosing and managing pregnancies complicated by cervical prolapse. With early recognition and appropriate conservative intervention, most dangers can be avoided. Obstetric backup should be available should serious complications arise.

\section{References}

1. Hill PS. Uterine prolapse complicating pregnancy. A case report. J Reprod Med 1984;29:631-3.

2. Klawans AH, Kanter AE. Prolapse of the uterus and pregnancy. Am J Obstet Gynecol 1949;57:939-46.

3. Kurzel RB, Nichols DH. Genital prolapse during pregnancy. J Reprod Med 1980;24:46-7.

4. Keettel WC. Prolapse of the uterus during pregnancy. Am J Obstet Gynecol 1941;42:121-6.

5. Kibel I. Pregnancy at term in prolapsed uterus. Am J Obstet Gynecol 1944;47:703-4.

6. Lavery JP, Sek-Boey C. Uterine prolapse with pregnancy. Obstet Gynecol 1973;42:681.

7. Tomezsko JE, Sand PK. Pregnancy and intercurrent diseases of the urogenital tract. Clin Perinatol 1997; 24:343-68. 
8. Piver MS, Spezia J. Uterine prolapse during pregnancy. Obstet Gynecol 1968;32:765-9.

9. van Dongen $L$. The anatomy of genital prolapse. $S$ Afr Med J 1981;60:357-9.

10. Brown HL. Cervical prolapse complicating pregnancy. J Natl Med Assoc 1997;89:346-8.

11. al-Qattan MM. Abdominoplasty in multiparous women with severe musculoaponeurotic laxity. $\mathrm{Br} \mathrm{J}$ Plast Surg 1997;50:450-5.

12. Gass ML. Does abdominoplasty predispose to pelvic relaxation. Plast Reconstr Surg 1990;86:1240-1.

13. Findley P. Prolapse of the uterus complicating pregnancy. JAMA 1911;57:2131.

14. Seitz L. Ber. u. d. ges. Gynak. u. Geburtsh 1901;6:244. 